\title{
Bayesian Approach for Distribution System State Estimation with non-Gaussian Uncertainty Models
}

\author{
Paolo Attilio Pegoraro, Member, IEEE, Andrea Angioni, Student Member, IEEE, Marco Pau, Member, IEEE, \\ Antonello Monti, Senior Member, IEEE, Carlo Muscas, Senior Member, IEEE, Ferdinanda Ponci, Senior \\ Member, IEEE, Sara Sulis, Member, IEEE
}

\begin{abstract}
To deal with the increasing complexity of distribution networks that are experiencing important changes, due to the widespread installation of Distributed Generation and the expected penetration of new energy resources, modern control applications must rely on an accurate picture of the grid status, given by the Distribution System State Estimator (DSSE). DSSE is required to integrate all the available information on loads and generators power exchanges (pseudomeasurements) with the realtime measurements available from the field. In most cases the statistical behavior of the measured and pseudomeasured quantities cannot be approximated by a Gaussian distribution. For this reason, it is necessary to design estimators that are able to use measurements and forecast data on power flows that can show a non-Gaussian behavior. In this paper, a DSSE algorithm based on Bayes's rule, conceived to perfectly match the uncertainty description of the available input information, is presented. The method is able to correctly handle the measurement uncertainty of conventional and synchronized measurements and to include possible correlation existing between the pseudomeasurements. Its applicability to medium voltage distribution networks and its advantages, in terms of accuracy of both estimated quantities and uncertainty intervals, is demonstrated.
\end{abstract}

Index Terms-Distribution System State Estimation, Bayesian Theory, Non-Gaussian Uncertainties, Measurement Correlation, Active Distribution Grids.

\section{INTRODUCTION}

In the smart grid scenario, the distribution grid will be the level of the electric system interested by the most important changes [1]. The massive installation of Distributed Generation (DG) already overturned the traditional view of distribution grids as simple passive networks, transforming them in active grids with bi-directional power flows. In future, the expected diffusion of electric vehicles, the use of storage devices and the increasing presence of power electronics will lead the distribution network to become a highly complex system [2]. To deal with such a high complexity, new control functionalities need to be developed. For an efficient management of

P. A. Pegoraro, C. Muscas, S. Sulis are with the Department of Electrical and Electronic Engineering of the University of Cagliari, Piazza d'Armi, 09123 Cagliari, Italy (email: [paolo.pegoraro, carlo, sara.sulis]@ diee.unica.it).

A. Angioni, M. Pau, A. Monti, F. Ponci are with the Institute for Automation of Complex Power Systems, RWTH Aachen University, Aachen 52062, Germany (email: [aangioni, mpau, amonti, fponci]@eonerc.rwth-aachen.de).

(c) 2017 IEEE. Personal use of this material is permitted. Permission from IEEE must be obtained for all other users, including reprinting/republishing this material for advertising or promotional purposes, creating new collective works for resale or redistribution to servers or lists, or reuse of any copyrighted components of this work in other works. DOI:10.1109/TIM.2017.2728398 Publisher version/http://ieeexplore.ieee.org/ stamp/stamp.jsp?tp=\&arnumber $=8008784 \&$ isnumber $=4407674$ the distribution system, these applications require an accurate monitoring of the operating conditions, which is provided by Distribution System State Estimation (DSSE) tools.

In spite of the significant efforts made in recent years to develop DSSE algorithms tailored to the specific characteristics of distribution grids [3]-[7], many challenges still prevent an easy deployment of DSSE on the field [8]. A comprehensive review of DSSE methodologies is available in [9].

One of the main issues is the lack of redundant measurements, which is a pre-requisite for the accurate and reliable operation of State Estimation (SE) algorithms [10]. The lack of a sufficient number of measurements is usually tackled by introducing the so-called pseudomeasurements, typically represented by forecast measurements about the power consumption or injection at the different nodes of the grid.

Despite being essential to reach the observability of the distribution network, pseudomeasurements bring some limitations to the accuracy performance achievable with the DSSE results, mainly for two reasons:

- since they are forecast measurements based on statistical and/or historical data, the confidence level on this information is generally low, and consequently they need to be considered with a very low accuracy; this is automatically reflected on the accuracy of the DSSE output [11];

- because of the particular behaviour of loads and DG (or their combination in case of prosumers), the probability distribution around the expected value assumed as pseudomeasurement can differ significantly from the Gaussian distribution commonly adopted in classic Weighted Least Squares (WLS) estimators; any approximation used to represent this uncertainty in the DSSE model translates in an error that propagates to the final DSSE results.

The pseudomeasurement characteristics have been analysed in different studies during last years. In [12], the impact of the smart metering data aggregation restriction on DSSE is considered. In [13] a procedure based on an Artificial Neural Network (ANN) has been proposed to extract pseudomeasurements and to enhance their reliability for DSSE purposes. An ANN has been also used in [14] to process the last SE results and to generate the pseudomeasurements accordingly. Other papers focused instead on the modelling of the distribution. In [15], different models, namely the normal, the log-normal and the beta distribution, have been tested to verify their suitability to represent load pseudomeasurements. The beta distribution was suggested as the best one, due to the possibility of tuning the parameters for obtaining the desired shape and skewness. 
Beta distribution has been also proposed and used in [16] to represent the uncertainty distribution of residential loads. Another interesting option proposed as an alternative is the use of Gaussian Mixture Models (GMM, see for example [17] and [18]), where the overall uncertainty distribution is obtained by means of a weighted sum of different Gaussian curves. This solution is appealing above all when bimodal uncertainty distributions exist, as it could be the case when nodes with prosumers or the combination of loads and DG are present. In [19], a framework is proposed to deal with non-Gaussian measurements when the mathematical model of the distribution is explicitly known, by translating them into an equivalent normal space by means of suitable transformations.

All the above mentioned studies indicate that the uncertainty distribution of the pseudomeasurements can vary significantly, depending on the characteristics of the loads or DG connected downstream the node and the particular scenario taken into account. For this reason, to maximize the DSSE performance, it is important to design flexible state estimators able to deal with any kind of uncertainty distribution. A first attempt to design a state estimator able to deal with different statistical distributions, based on the Bayesian theory, has been discussed in [20]. While the Bayes' rule has been applied in some fields related to power systems, like in topology identification [21], its application as a paradigm for state estimation is a novelty proposed by the authors. In [20], a preliminary investigation was carried out to gain a first idea on the potential benefits of a Bayesian approach in a state estimation process, but several simplifying assumptions were present. First of all, all the pseudomeasurements were assumed to be not correlated between each other. Then, only Gaussian distributions were assumed for the real measurements taken from the field. Finally, only traditional power, voltage and current measurements were considered. The estimator was tested on a small LV grid to show the possible advantages it offers with respect to classical solutions based on a WLS approach.

Starting from the encouraging outcomes of [20], in this paper, the technique has been improved and generalized by removing all the above simplifications and by keeping into account additional important aspects of the statistical description of measurements and pseudomeasurements. In particular, the refined technique presented in this paper also allows including the possible correlations existing between pseudomeasurements, like those between active and reactive power at a given node, and considers a more general model of the quantities provided by the measurement instruments, which can reflect any generic uncertainty distribution. With respect to previous literature, the proposal here presented thus allows dealing with measurements and prior information having any uncertainty distribution, also including empirical distributions that cannot be classified under a specific non-Gaussian model. In addition, the estimator is further refined to include synchronized measurements from Phasor Measurement Units (PMUs). The newly designed estimator is then tested on a sample MV grid, using statistical models gathered from real data to define the pseudomeasurements, with different types of loads and generators and different power profiles. The final outcome of this paper is thus the proposal of a general paradigm for DSSE that is applicable to both MV and LV networks and allows including even complex statistical models of measurements, loads and generators, overcoming the main issues of classic simplified or model-based approaches.

In the following, the paper is structured as follows. In Section II. the mathematical model used to design the Bayesian estimator is presented. Section III describes the details about the considered power profiles, showing in particular the statistical distributions achieved from the gathered real data. In Section IV] the proposed Bayesian DSSE is tested and the obtained results are discussed. Finally, Section $\mathrm{V}$ summarizes the characteristics and peculiarities of the proposed approach and concludes the paper.

\section{PRoposed DSSE BAyesian ESTIMATOR}

The proposed estimator exploits the classical DSSE measurement model:

$$
\mathbf{z}=\mathbf{h}(\mathbf{x})+\mathbf{e}
$$

where $\mathbf{z}$ is the $M$-dimensional vector of measurements gathered from the field, $\mathbf{x}$ is the $N$-dimensional vector of state variables, $\mathbf{h}$ is the vector of measurement functions, defining the relationship between state variables and measured quantities and $\mathbf{e}$ is the vector of measurement errors. Each measurement $z_{i}$ has its own probability density function (PDF) $f_{z_{i}}(\cdot)$ that reflects the probability distribution that can be associated to the measurement. The choice of the PDF to be assumed depends on the information available from characterization processes or from the instruments specifications [22]. When further or specific information is missing, as it is common when dealing with instrument datasheets, uniformity should be assumed with a value range defined by the measurement accuracy. In this case, the PDF of the corresponding measurement error $e_{i}$ becomes:

$$
f_{e_{i}}(a)= \begin{cases}\frac{1}{2 \Delta z_{i}}, & \forall a \in\left(-\Delta z_{i},+\Delta z_{i}\right) \\ 0, & \text { otherwise }\end{cases}
$$

where $\Delta z_{i}$ and $-\Delta z_{i}$ are the upper and lower limit of the error interval. Instead, if normality is considered, the following holds:

$$
f_{e_{i}}(a)=\frac{1}{\sqrt{2 \pi} \sigma_{z_{i}}} e^{-\frac{a^{2}}{2 \sigma_{z_{i}}}}
$$

where $\sigma_{z_{i}}=\sigma_{e_{i}}$ is the standard deviation of $z_{i}$. If not directly available, $\sigma_{z_{i}}$ is typically chosen as a fraction of the $\Delta z_{i}$ (depending on the assumed coverage factor).

\section{A. Bayes Approach for DSSE}

With a Bayesian approach, the estimated state $\hat{\mathbf{x}}$ can be obtained from the posterior distribution, given the measurement vector and the prior distribution of the state. In this context, the estimation is obtained as the expectation of the posterior distribution, given the measurements, as follows:

$$
\hat{\mathbf{x}}=\mathbb{E}[\mathbf{x} \mid \mathbf{z}]=\iint \mathbf{a} f_{\mathbf{x} \mid \mathbf{z}}(\mathbf{a} \mid \mathbf{z}) d \mathbf{a}
$$

where the multidimensional integral is performed in the whole state space and a represents the integration variable that spans 
the values $\mathbf{x}$ can assume, see [23]. Following the Bayes paradigm, the posterior is built as follows:

$$
f_{\mathbf{x} \mid \mathbf{z}}=\frac{f_{\mathbf{z} \mid \mathbf{x}} f_{\mathbf{x}}}{f_{\mathbf{z}}}
$$

where $f_{\mathbf{x}}$ is the prior PDF of the state variables and $f_{\mathbf{z} \mid \mathbf{x}}$ is the conditional PDF of the measurements, that becomes the likelihood function of the state given the measurements set. The measurement vector can include conventional measurements as voltage amplitude, current amplitude or power (active and reactive branch or node powers) measurements, or phasor measurements obtained by phasor measurement units (PMUs) synchronized with respect to a coordinated universal time reference. In the DSSE, due to the scarcity of realtime measurements, prior information on loads and generators power becomes necessary to obtain the full observability of the state. This data are usually used in the DSSE as pseudomeasurements to which a large uncertainty is associated.

The statistical description of the absorbed (or generated) powers can be usually extracted by the historical data and exploited to integrate the inputs of the DSSE. The underlying probability distribution can be non-Gaussian, also depending on the type of the loads and generators, on the time resolution of the available statistical information and on the degree of aggregation of the adopted model. This observation, together with the possible non-gaussianity of the measurements, is the main reason to design an estimation algorithm based on Bayes theory, which allows the state to be estimated considering the full statistical description of the measurements and pseudomeasurements.

The proposed DSSE approach directly applies the Bayes' rule (5), by considering as state vector the vector of power injections along with a voltage node phasor (the slack bus is chosen). Thus the state $\mathrm{x}$ can be defined as follows:

$$
\mathbf{x}=\left[\begin{array}{c}
V_{s} \\
\varphi_{s} \\
\mathbf{P} \\
\mathbf{Q}
\end{array}\right]
$$

where $\mathbf{P}$ and $\mathbf{Q}$ are the active and reactive node power balance vectors, while $V_{s}$ and $\varphi_{s}$ are the voltage magnitude and phase angle of the chosen reference node. If at least one PMU measurement is available, the absolute phase angle can be measured [5]. Moreover, as shown in [24], if at least two PMUs are present, adding a reference phase angle in the state allows also to improve the estimation of the absolute phase angle profile beyond the accuracy of PMUs. Otherwise, only phase angle differences can be evaluated and $\varphi_{s}$ should be removed from the state vector.

The DSSE formulation requires the definition of the elements in (5). The prior $f_{\mathbf{x}}$ can be obtained by the statistical knowledge of $\mathbf{P}$ and $\mathbf{Q}$ and by typical assumptions on slack bus voltage. In particular, the PDFs of active and reactive powers can be derived by a model matching with known distributions or in an empirical way, by histograms or empirical distribution fitting (kernel based for instance). The prior $f_{V_{s}}$ of $V_{s}$ can be easily assumed as uniform in a given interval $(1 \pm 0.1$ p.u., for instance).
In practice, when PMUs are available, the substation bus is usually monitored and the corresponding phase angle can be chosen as a reference: a uniform $\operatorname{PDF} f_{\varphi_{s}}$ around the measured value can thus be adopted to define the prior considering the maximum PMU phase angle deviation. In all the other cases, without any loss of generality and recalling that phase angle differences are low in distribution networks, a guess interval can be used to define the prior around the nearest PMU measurement.

The likelihood function is instead built from the information available on the measurement accuracy. In [20] the conditional PDF was assumed to be the product of $M$ independent Gaussians (one for each measurement) with standard deviations chosen as one-third of the corresponding maximum deviations.

However, if only accuracy specifications reported in instrument datasheets are available, the assumption of uniformly distributed measurements is more reasonable. Assuming independence among measurements, it is thus possible to express the conditional $\operatorname{PDF} f_{\mathbf{z} \mid \mathbf{x}}$ as follows:

$$
\begin{aligned}
& f_{\mathbf{z} \mid \mathbf{x}}(\mathbf{b} \mid \mathbf{a})=\prod_{i=1}^{M} f_{z_{i} \mid \mathbf{x}}\left(b_{i} \mid \mathbf{a}\right)= \\
& \begin{cases}\frac{1}{\prod_{i=1}^{M} 2 \Delta z_{i}}, & \forall \mathbf{b} \in \prod_{i=1}^{M}\left[h_{i}(\mathbf{a})-\Delta z_{i}, h_{i}(\mathbf{a})+\Delta z_{i}\right] \\
0, & \text { otherwise }\end{cases}
\end{aligned}
$$

where $\mathbf{b}$ is the generic point of the space and $h_{i}(\cdot)$ is the measurement function of the $i$-th measurement ( $i$-th component of $\mathbf{h}(\cdot))$. As described in Section II-D on numerical integration, to solve (4), several candidates a have to be computed for the state $\mathrm{x}$ and, for each point, the corresponding measurement vector $\mathbf{b}$ is computed. If the state is defined as in (6) all the needed quantities can be derived by means of powerflow calculation and all the measurement values $b_{i}$ to be used in (7) can thus be obtained.

Once $f_{\mathbf{x}}$ and $f_{\mathbf{z} \mid \mathbf{x}}$ are computed, the posterior of the state with respect to the given measurement set is obtained up to a multiplicative factor. The numerator in (5) is calculated, whereas the denominator, represented by the integration of the numerator in the whole state space, is not needed for the numerical solution of the DSSE estimation, as it is better described in Section III-D. The denominator, which represents the overall probability of the measurements, is a constant and thus does not affect the space exploration performed to achieve the estimated state.

\section{B. Handling of PMU Measurements}

PMUs measure amplitude and phase angles of voltage and current phasors. Depending on the specifications given in the device datasheet by the PMU manifacturer, two typical expressions of the measurement accuracy can be found: amplitude maximum deviation percentage (Max $\mathrm{AE} \%$ ) and maximum phase angle deviation (Max PE), or maximum percent Total Vector Error (TVE \%).

In the first case, for a generic measured voltage phasor $\bar{v}=V e^{j \varphi_{v}}$, amplitude and phase angle measurements can be inserted as uniform random variables as in 2, where $\Delta z_{V}=\frac{V \cdot \operatorname{Max} \mathrm{AE} \%}{100}$ and $\Delta z_{\varphi_{v}}=$ Max PE, respectively. 
Current phasors can be handled analogously. This approach is similar to that usually applied when the PMU measurements are included in classical WLS DSSE, depending also on the assumed probability distribution.

In the second case, when only the maximum TVE $\%$ is known, the proposed algorithm allows a phasor measurement handling that directly reflects the available information about measurement uncertainty. In fact, it is possible to directly define the joint PDF of the amplitude and phase angle given the state $\mathbf{x}$ (discussing only the voltage phasor measurement for the sake of simplicity), as follows:

$$
\begin{aligned}
f_{z_{V}, z_{\varphi_{v}} \mid \mathbf{x}}(\bar{b} \mid \mathbf{a})= & f_{z_{V}, z_{\varphi_{v}} \mid \mathbf{x}}\left(B, \varphi_{b} \mid \mathbf{a}\right)= \\
& \begin{cases}\frac{1}{\pi \Delta z_{V}{ }^{2}}, & \forall \bar{b} \in\left|\bar{b}-\bar{h}_{v}(\mathbf{x})\right| \leq \Delta z_{V} \\
0, & \text { otherwise }\end{cases}
\end{aligned}
$$

where $z_{V}$ and $z_{\varphi_{v}}$ stands for the corresponding amplitude and phase angle measurements, $\bar{b}=B e^{j \varphi_{b}}$ is the generic phasor in the plane, $\bar{h}_{v}(\cdot)$ is the complex measurement function of $\bar{v}$ and the bivariate PDF clearly defines a region of uniform probability describing a circle of radius equal to the maximum vector error around the measured phasor.

\section{Prior Definition in presence of Correlation between Active and Reactive Power}

From the statistics of the absorbed/generated active and reactive powers from loads/generators, analytical models or empirical distributions can be obtained and thus the priors computed for each node. In [20], active and reactive power injections/absorptions at a given node $i$ (that is $P_{i}$ and $Q_{i}$ ) were assumed uncorrelated, but, under real network operation, it is often useful to consider also correlation in the probabilistic model of the corresponding prior.

For this reason, in this paper, for each node $i$, the PDFs $f_{P_{i}}$ and $f_{Q_{i}}$, obtained from historical and statistical data, are considered as the marginals of the two-dimensional distribution of the node power balance. The correlation is introduced in the model of each $P_{i}, Q_{i}$ couple by means of a bi-dimensional copula. For each node $i$, the copula $C_{i}$ associated to the two random variables $P_{i}$ and $Q_{i}$ is a bi-variate random vector and can be defined, for instance, by its joint cumulative distribution function:

$$
F_{C_{i}}\left(u_{1}, u_{2}\right)=\mathbb{P}\left[U_{P_{i}} \leq u_{1}, U_{Q_{i}} \leq u_{2}\right]
$$

where $U_{P_{i}}=F_{P_{i}}\left(P_{i}\right)$ and $U_{Q_{i}}=F_{Q_{i}}\left(Q_{i}\right)$ are the two random variables obtained by transforming $P_{i}$ and $Q_{i}$ by means of their cumulative distribution functions. With such transformations, $U_{P_{i}}$ and $U_{Q_{i}}$ become uniform variables in the interval $(0,1)$ and $F_{C_{i}}$ allows to express the correlation between the active and reactive powers. In fact, Sklar's Theorem ([25]) guarantees that any multivariate joint distribution can be written in terms of univariate marginal distribution functions and a copula which describes the dependence structure between the variables. As a consequence, it is possible to decouple the prior definition problem into power marginals fitting and copula modeling. The bi-variate prior corresponding to the node pseudomeasurements can thus be computed in a generic point $\left(p_{i}, q_{i}\right)$ as:

$$
f_{P_{i}, Q_{i}}\left(p_{i}, q_{i}\right)=f_{C_{i}}\left(F_{P_{i}}\left(p_{i}\right), F_{Q_{i}}\left(q_{i}\right)\right) \cdot f_{P_{i}}\left(p_{i}\right) \cdot f_{Q_{i}}\left(q_{i}\right)
$$

where $f_{C_{i}}$ indicates the copula PDF. In this paper, without loss a generality, a simple Gaussian copula has been used for the tests to keep correlation into account (see Sections III] and IV].

Once the node prior is defined, the overall prior PDF of the state can be computed as follows:

$$
f_{\mathbf{x}}(\mathbf{a})=f_{V_{s}}\left(a_{1}\right) f_{\varphi_{s}}\left(a_{2}\right) \prod_{i=2}^{N_{\text {bus }}} f_{P_{i}, Q_{i}}\left(a_{i+1}, a_{i+N_{\text {bus }}}\right)
$$

where the vector a is arranged as in (6) and $N_{b u s}$ indicates the number of buses corresponding to loads or generators. The expressed formulation is general, but can be easily adapted to the case of zero-injection nodes. It is sufficient to reduce the state, and to substitute the corresponding state values with zero constants. This is particularly interesting in the context of medium voltage networks, in which there are often several zero-injection constraints. In the case of traditional measurements only, as aforementioned, the $\varphi_{s}$ is not included in the state vector and, correspondingly, $f_{\varphi_{s}}$ is dropped from (11).

\section{Numerical integration of Bayes estimation}

The solution of (4) and thus of the DSSE is performed by a numerical evaluation, using a Metropolis-Hastings $(\mathrm{M}-\mathrm{H})$ algorithm as in [20]. The method allows to obtain a sequence of points $\mathbf{x}$, belonging to the state space, with the statistical properties described by the target distribution $\Gamma(\mathbf{x})=f_{\mathbf{x} \mid \mathbf{z}}$.

The M-H algorithm works iteratively building the chain of the states $\mathbf{x}_{k}$ with simple rules. From an initial guess of the actual state, at each iteration $k$, using a proposal transition PDF $\Pi\left(\mathbf{d} \mid \mathbf{x}_{k}\right)$, a new proposal state point $\mathbf{y}_{k+1}$ is extracted and acts as a candidate for the next point $\mathbf{x}_{k+1}$ determined with the following decision step:

$$
\mathbf{x}_{k+1}= \begin{cases}\mathbf{y}_{k+1}, & \text { with a given probability } \alpha\left(\mathbf{y}_{k+1} \mid \mathbf{x}_{k}\right) \\ \mathbf{x}_{k}, & \text { otherwise }\end{cases}
$$

where $\alpha$ is the acceptance probability that, when the proposal distribution is symmetric $\left(\Pi\left(\mathbf{x}_{k} \mid \mathbf{y}_{k+1}\right)=\Pi\left(\mathbf{y}_{\mathbf{k}+\mathbf{1}} \mid \mathbf{x}_{k}\right)\right)$, can be expressed as follows:

$$
\alpha\left(\mathbf{y}_{k+1} \mid \mathbf{x}_{k}\right)=\min \left\{1, \frac{\Gamma\left(\mathbf{y}_{k+1}\right)}{\Gamma\left(\mathbf{x}_{k}\right)}\right\}
$$

As a consequence, $\alpha\left(\mathbf{y}_{k+1} \mid \mathbf{x}_{k}\right)$ is one when $\Gamma\left(\mathbf{y}_{k+1}\right) \geq \Gamma\left(\mathbf{x}_{k}\right)$ (the ratio of the two probability densities is greater than one, thus meaning an increase in the probability is achieved) and the new point is always kept, otherwise it is accepted with a probability equal to the relative decrease of the target PDF when passing from the current point to the proposed one.

To make the numerical computation more stable and the Markov chain definition more effective, all the computations concerning the PDFs are here performed by means of logarithms (using the so-called $\operatorname{logPDF}$ functions, $\log f$ in the 
following for brevity). With this choice, $\mathbf{y}_{k+1}$ is accepted if the logarithm of a random variable $u$, extracted from a uniform distribution on the interval $(0,1)$, is less than $\log \left(\alpha\left(\mathbf{y}_{k+1} \mid \mathbf{x}_{k}\right)\right)$ (or always accepted if $\alpha\left(\mathbf{y}_{k+1} \mid \mathbf{x}_{k}\right)=1$ ). Recalling the definition of $\Gamma$ in the specific case, from (7) and (11) the following expression is obtained while computing $\alpha$ :

$$
\begin{gathered}
\log \left(\alpha\left(y_{k+1} \mid x_{k}\right)\right)=\min \{1 \\
\sum_{i=1}^{M}\left[\log f_{z_{i} \mid \mathbf{x}}\left(\mathbf{y}_{k+1}\right)-\log f_{z_{i} \mid \mathbf{x}}\left(\mathbf{x}_{k}\right)\right] \\
\quad+\log f_{V_{s}}\left(y_{k+1,1}\right)-\log f_{V_{s}}\left(x_{k, 1}\right) \\
\quad+\log f_{\varphi_{s}}\left(y_{k+1,2}\right)-\log f_{\varphi_{s}}\left(x_{k, 2}\right) \\
+\sum_{i=2}^{N_{\text {bus }}}\left[\log f_{C_{i}}\left(F_{P_{i}}\left(y_{k+1, i}\right), F_{Q_{i}}\left(y_{k+1, i+N_{\text {bus }}}\right)\right)\right. \\
\quad-\log f_{C_{i}}\left(F_{P_{i}}\left(x_{k, i}\right), F_{Q_{i}}\left(x_{k, i+N_{\text {bus }}}\right)\right) \\
\quad+\log f_{P_{i}}\left(y_{k+1, i}\right)-\log f_{P_{i}}\left(x_{k, i}\right) \\
\left.\left.+\log f_{Q_{i}}\left(y_{k+1, i+N_{\text {bus }}}\right)-\log f_{Q_{i}}\left(x_{k, i+N_{\text {bus }}}\right)\right]\right\}
\end{gathered}
$$

where the same state as in (6) is assumed and the second subscript index for $y_{k+1, i}$ (and analogously for the other variables) indicates the element position in the vector $\mathbf{y}_{k+1}$.

Under a few constraints, the obtained chain converges to the desired posterior distribution, and is ergodic. To allow a faster exploration of the state space, an adaptive proposal distribution is used as in [20] and a custom tailored version of the algorithm in [26] has been chosen. Once $n$ points in the state space are obtained, the DSSE estimation is given by their average (excluding the first initial burn-in period). Due to the mentioned properties of the extracted samples, the obtained average is the numerical computation of (4) and thus represents the estimation of the expected state given the prior and measurement information. With simple statistical estimators, quantiles of the posterior can be also computed for each component of the state to estimate the uncertainty intervals to be associated to the estimated quantities.

\section{Statistical Analysis of Power Profiles}

The DSSE has to be designed to use, besides SCADA measurements and, as increasingly expected in the near future, synchrophasors at primary and secondary substations, also active and reactive power information at the nodes. In case of LV systems, the smart meters, connected at the terminals of loads and/or distributed generators (DGs), can help collecting data on the behavior of the loads/generators in terms of active and reactive power injections. In [20] it was shown that, in the LV case, single electrical users, both residential and small commercial industrial entities, have peculiar power distributions, much different from normality, thus taking significant advantages from the application of a Bayesian approach.

In this paper the application to MV grids is specifically explored, in order to reinforce the advantages and the applicability of the proposed method. At this higher voltage level, the relevance of the Bayes approach could be questioned by considering that the aggregation of several LV customers with limited power consumption might give rise to distributions much closer to the Gaussian PDF. Actually, in MV networks, the aggregation of small LV customers coexist with large commercial and industrial customers. Therefore, the MV loads can also show strong non-Gaussian power profiles, due to peculiar features of load units. Besides, the DGs often have non-normal statistical features, thus making the scenario even more interesting. As recalled in Section II-A the choice of the model must be made on a case by case basis, since the type of loads, the level of aggregation and of profile information may influence the decision.

Power measurements in MV nodes can be directly integrated in the monitoring system and DSSE architecture. When this does not happen, such power measurements can be used to get a statistical knowledge of the users behavior. This information represents the pseudomeasurements of the DSSE and is traditionally provided by assigning an expected value and a standard deviation to the forecast node power balance at a given time point. In the proposed Bayes approach, the pseudomeasurements are directly represented by the PDFs built from the collected data and the models of loads and generators. In a real application, power measurements can be performed in a subset of the electricity users [11] to gather the statistical information on the exchanged power consumptions/generations and build the pseudomeasurements for all the remaining users, as done in [20] for LV networks.

For this paper, sample statistical resources for MV loads have been obtained from the OpenEI energy information repository [27] for the state of California in US. Specifically, profiles with one hour time resolution have been extracted for multi-family residential buildings, supermarkets and primary schools. Similarly, power profiles, with one hour time resolution, for PV power plants were extracted from the PVWatts Calculator tool [28] based on historical irradiance data, from the state of California in US. Comparing different daily power profiles suggested that, in each time instant the power generation or consumption (or, alternatively, the difference of the two, if they are related to a single MV node) can have a non-Gaussian probability distribution, whose pattern should be evaluated for proper integration into the DSSE as pseudomeasurement. The patterns can be usually differentiated for loads among seasons (winter, middle season, summer), weekdays (working days, Saturdays and Sundays) and, of course, time of the day (that is associated to a given hour). For PV generation, the statistics can be differentiated among seasons and time of the day.

The histograms for the loads are presented in Fig. 1. in terms of relative frequency of occurrence, for a typical supermarket profile (with size and equipment as defined in [27]) for three different hours of a typical mid-season (spring-autumn) working day. In Fig. 2 a power consumption histogram is presented for winter, mid-season and summer, given the same time of the day (13:00). It can be observed that the load absorption shows to be strictly non-Gaussian. Furthermore, changes with time of the day and season do not only impact the average power consumption but also the shape of the distribution, and thus the uncertainty of the corresponding pseudomeasurement.

Further observations may be done based on the histogram 


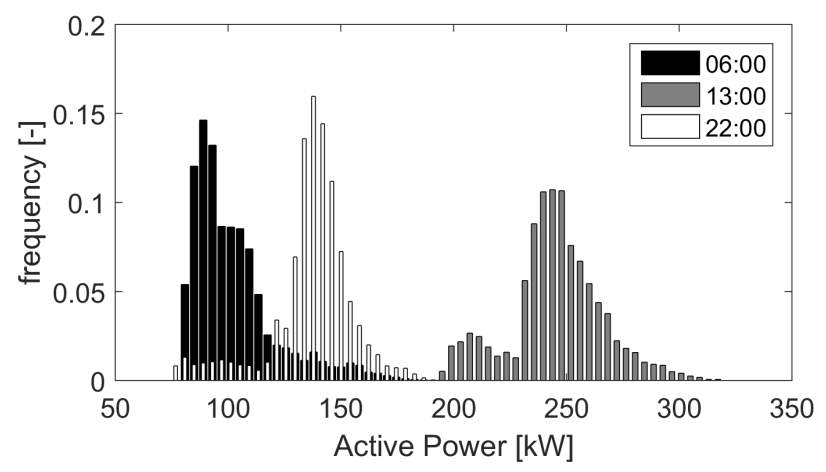

Fig. 1. Comparison of load power consumption histograms of a typical supermarket at 06:00 and 13:00 and 22:00 of a typical working day of mid season.

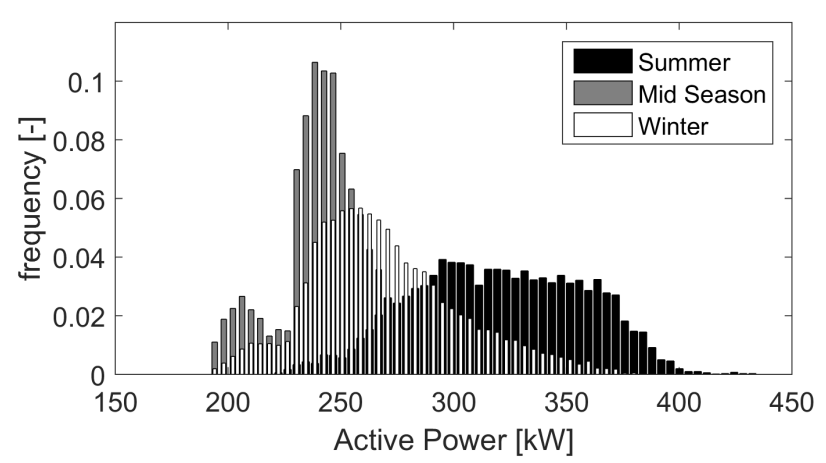

Fig. 2. Comparison of load power consumption histograms of a typical supermarket at 13:00 between summer, mid-season and winter working days.

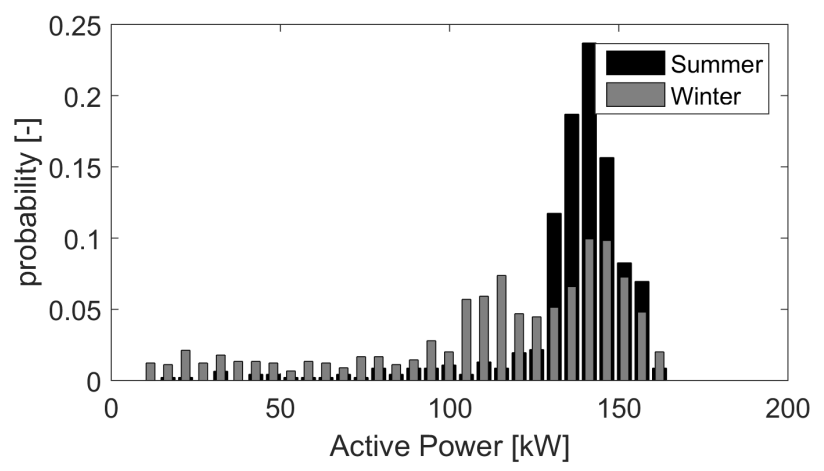

Fig. 3. Comparison of PV power production histograms at 13:00 between summer and winter days.

of power generation valid for a standard PV plant of $200 \mathrm{~kW}$, therefore suitable for installation at MV level. In Fig. 3, the histogram for PV power production in winter and summer at 13:00 are compared. In Fig. 4, the PV histograms are plotted for two different times of the day (09:00 and 15:00) of a typical mid-season day. It can be noticed that, as expected, both season and hour strongly impact on the probability distribution of the generated power. Anyway, for such a type of plant, even if the PDF can change, resulting in shape modification and average shifting, it is clearly non-Gaussian. For this reason, a correct modeling of DG behavior is essential to build reliable pseudomeasurements.

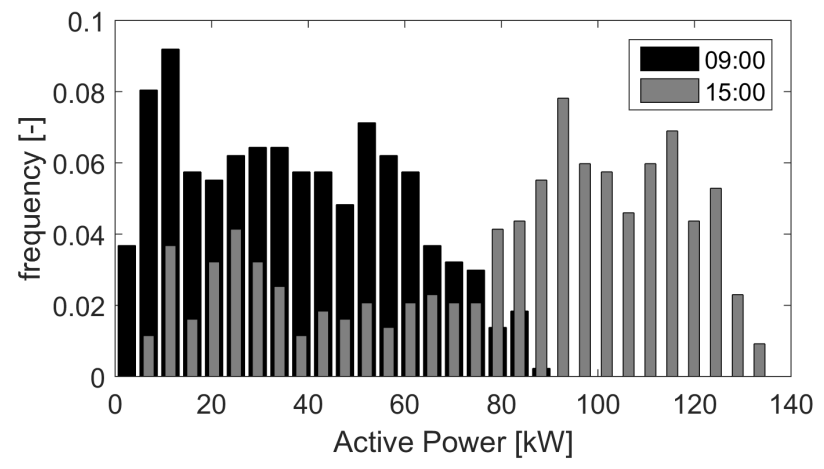

Fig. 4. Comparison of PV power production histograms at 09:00 and 15:00 of a typical mid-season day.

Beside the presented statistics, it is also important to give a representation of the correlation, in particular between active and reactive power at a given node. In this paper, to test the features of the proposed algorithm, the presence of correlation is simulated by defining the power factors of the given loads. In particular, the $\cos \phi$ is assumed to be a uniformly distributed random variable in a given interval (in the tests a range $0.95-1$ is considered) and to be independent from the active power. With such a rule, for each active power value, the corresponding reactive power is extracted, thus building a full dataset and giving a realistic relationship between $P_{i}$ and $Q_{i}$ (for the generic node $i$ ) to be used in the prior modeling. In the following, the method described in Section II-C is adopted to include correlation, while the kernel distribution fitting with Epanechnikov kernel is used to define the marginal PDFs.

All the above considerations build the groundwork for the study on the impact of prior definitions on the DSSE estimation uncertainty in the next section.

\section{TESTS AND RESUlTS}

In the following, a $20 \mathrm{kV} \mathrm{MV}$ sample network is used to discuss the performed tests and highlight the characteristics of the proposed DSSE. The network has six buses along one feeder (see Fig. 57): the first one corresponds to the HV/MV substation, while the others are connected to MV loads that combine absorption and generation as reported in Table I Since it is a simple network for illustrative purposes, all the line impedances have been assumed equal to $0.7031+$ $j 0.141 \Omega / \mathrm{km}$ (corresponding to a cable OC_AL_50_169 from Atlantide project [29]) and the line length is assumed equal to $0.8 \mathrm{~km}$ for each branch.

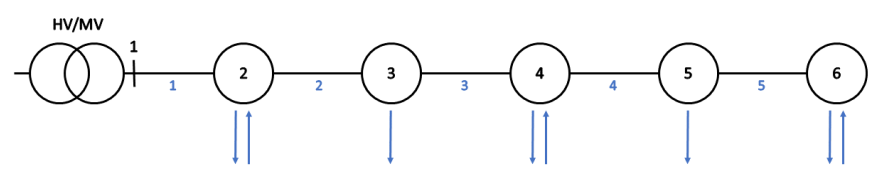

Fig. 5. Topology of the sample MV test grid. 
TABLE I

TYPE OF LOADS AND GENERATORS CONNECTED TO EACH BUS

\begin{tabular}{|c|c|c|c|c|c|}
\hline Node & 2 & 3 & 4 & 5 & 6 \\
\hline \hline Customers & apartments & supermarket & primary school & apartments & apartments \\
\hline Generators & PV & - & PV & - & PV \\
\hline
\end{tabular}

Both non-synchronized and synchronized measurements have been considered. Voltage amplitudes, active and reactive power flows, and voltage and current phasors are considered in the following. Measurements are assumed to be uniformly distributed in the interval defined by the accuracy of the instruments. In particular, $\pm 1 \%$ for voltage magnitude and $\pm 3 \%$ for active and reactive power flow measurements are employed. For synchronized measurements, $\pm 0.7 \%$ and $\pm 0.7 \cdot 10^{-2} \mathrm{rad}$ are assumed, respectively, for amplitude and phase angle PMU measurements.

As described in Section III] the load and generator priors can be referred to a given hour and season. All the reported results in the following correspond to the time of the day 10:00 of a generic mid-season day.

Since the proposed estimator is conceived to keep into account a better model of pseudomeasurements, it is particularly interesting to check its behavior in terms of power estimations. The knowledge of power injections from DSSE is crucial, for instance, for distribution management system applications (DMS).

In a first test, as a counter-check of the validity of the Bayesian approach, the DSSE is performed when only one voltage magnitude measurement is available at the substation. This means that no information is given other than pseudomeasurements, that is prior knowledge on the nodes power absorption/injection.

In Figs. 6(a) and 6(b), as an example, the posterior PDFs obtained from the points produced by the $\mathrm{M}-\mathrm{H}$ algorithm chain for the active powers of nodes 3 and $4\left(P_{3}\right.$ and $\left.P_{4}\right)$ are reported along with their prior PDFs. The posterior, as for the prior, has been obtained by kernel fitting from the data. As expected, the two curves are very similar, confirming that the method is able to reproduce the statistical behavior of the data and to give as output a representative population. Besides, the capability of matching a non-Gaussian distribution suggests the advantages of the method when generic loads/generators are present.

The inclusion of the correlation, following the approach based on copulas and described in Section [II-C, allows to match the dependency of active and reactive power for each node. As an example, for node 4 , the correlation coefficient $\rho_{P_{4} Q_{4}}$ of the generated data is 0.87 , very similar to 0.88 , which is the correlation coefficient of the original $P_{4}$ and $Q_{4}$ data, whereas it reduces to less than $10^{-3}$ if no correlation modeling is used. Fig. 7 graphically shows how the correlation between active and reactive power is reproduced.

Once the capability of the method to comply with a given statistical description has been confirmed by the above preliminary test, it is then possible to test its behaviour with different measurement configurations. In particular, Figs. 8(a) and 8(b) report the power injection estimation results when two voltage measurements (nodes 1 and 5) and two active and reactive

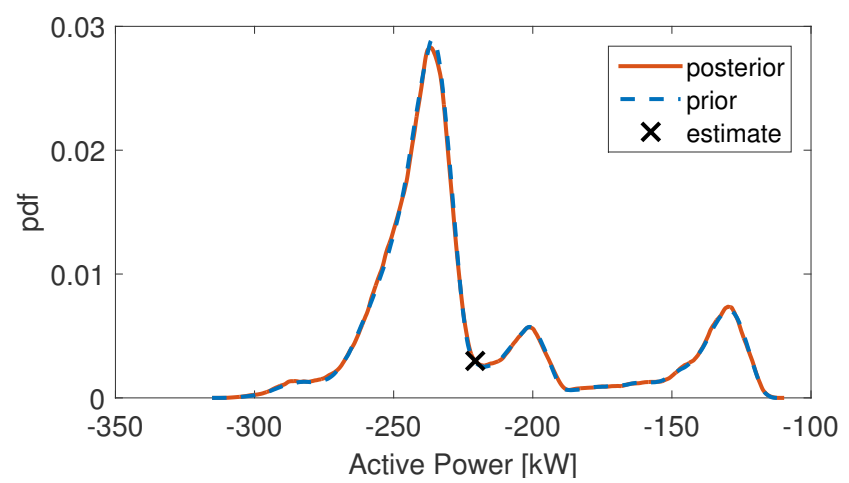

a)

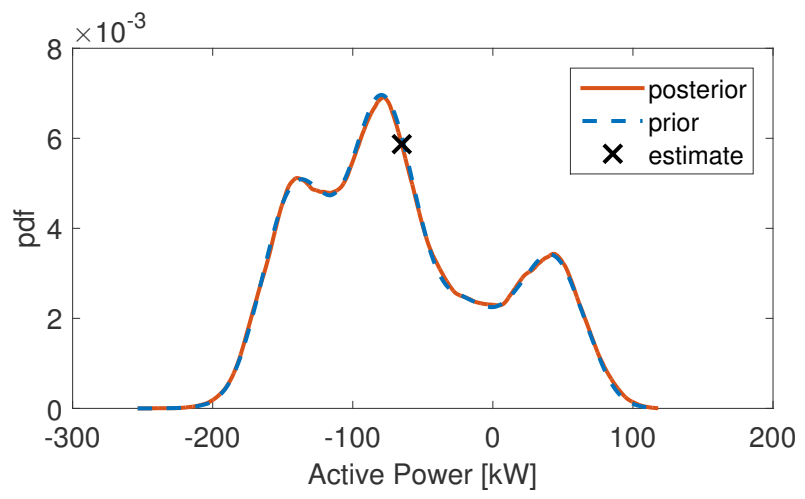

b)

Fig. 6. Active power prior and posterior with only one voltage measurement: a) node 3 ; b) node 4
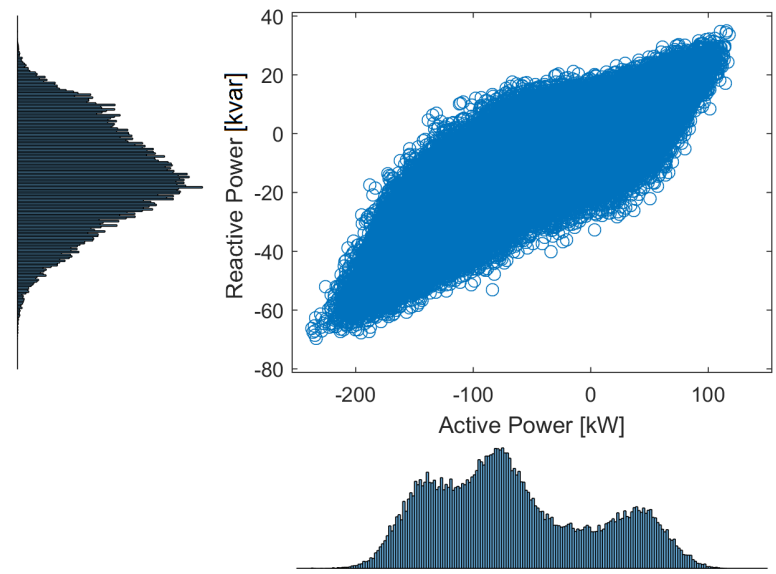

Fig. 7. Active and reactive power injection scatter plot

power flow measurements (branches 1 and 3) are used. It is clear how the impact of the measurements and, in particular, of power flow ones, makes the posterior narrower, as expected due the additional available information. Thus, the real-time data help, during the estimation process, to neglect regions that where suggested by the priors but are not supported by actual measurement information. The posterior are still non-Gaussian and the uncertainty intervals are non-symmetric around the estimated values.

Focusing on the estimation accuracy, Table III shows the 


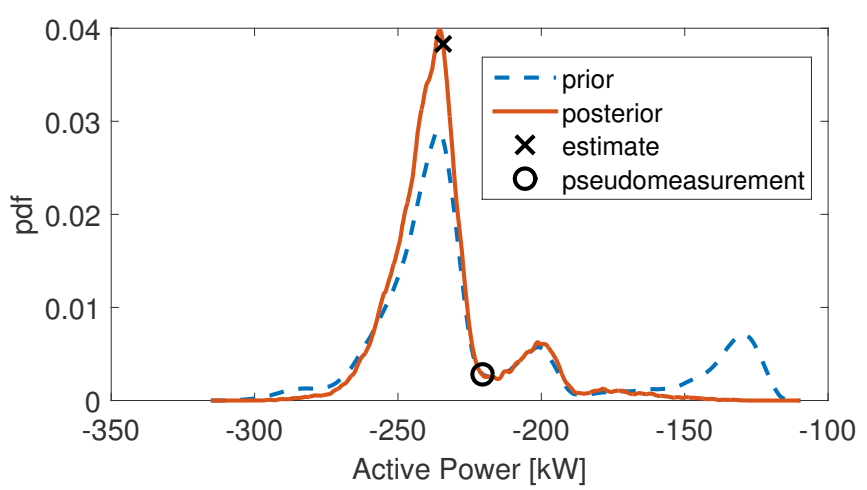

a)

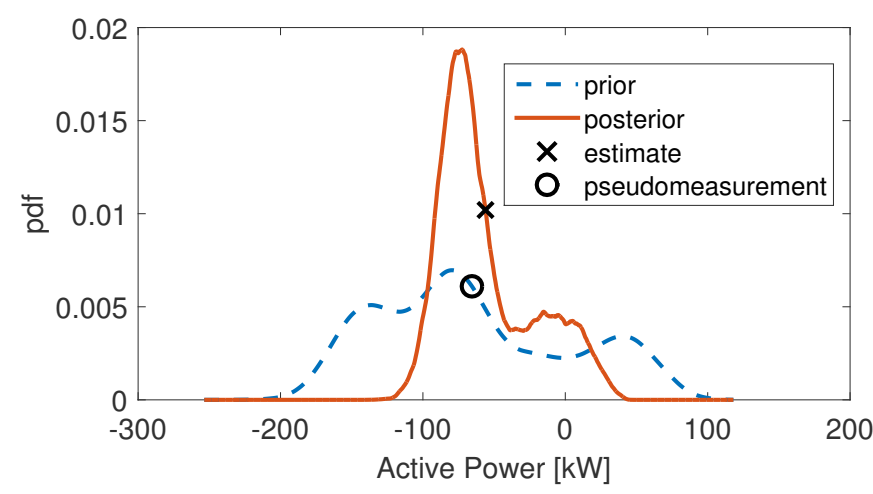

b)

Fig. 8. Active power prior and posterior with voltage and powerflow measurements: a) node 3 ; b) node 4

results in terms of Root Mean Square Errors (RMSEs) for 300 Monte Carlo trials when correlation is considered or not. It is clear that, beside the advantages in the modeling, the estimation accuracy is enhanced, except for node 5 , which is characterized by low power and power dispersion, and by low correlation.

TABLE II

RMSE OF POWER INJECTION ESTIMATIONS WHEN CORRELATION IS INCLUDED IN THE MODEL OR NOT

\begin{tabular}{|c|c|c|c|}
\hline \multirow{2}{*}{ Node } & \multicolumn{2}{|c|}{ RMSE [kW] } & \multirow{2}{*}{ Decrease [\%] } \\
\cline { 2 - 3 } & no correlation & correlation & \\
\hline \hline 2 & 22.1 & 21.5 & -3 \\
3 & 21.8 & 21.1 & -3 \\
4 & 30.6 & 29.3 & -4 \\
5 & 1.7 & 1.7 & 0 \\
6 & 30.5 & 29.3 & -4 \\
\hline
\end{tabular}

Further tests have been performed considering synchronized measurements instead of conventional ones, and, in particular, current phasors are used instead of power flow measurements. For the aforementioned reasons, it is important to check the DSSE performance in terms of power estimation also in the case of a PMU-based measurement system. Fig. 9 shows an example of the estimated node powers along with their estimated expanded uncertainty intervals, compared with the

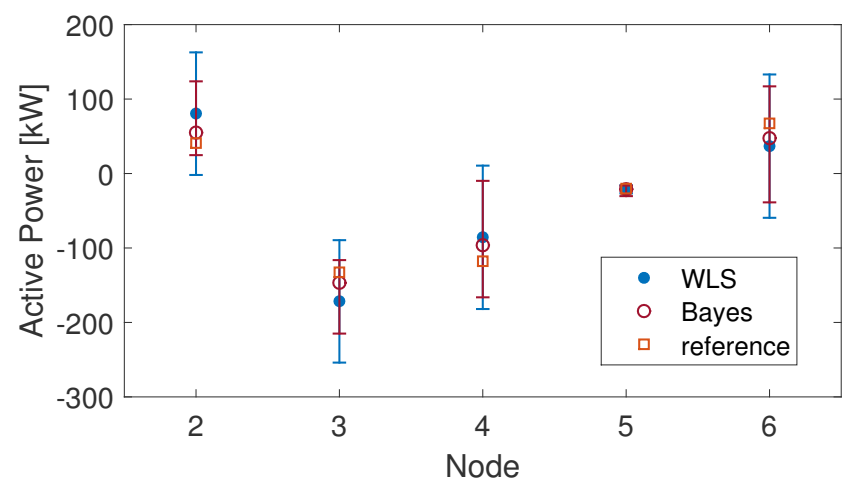

Fig. 9. Active power estimations with their estimated uncertainties: comparison of the Bayes and WLS approaches

WLS outcomes. The WLS is used as a term of comparison because it is the most widespread technique nowadays in DSSE and it gives the Bayesian estimator when measurements and pseudomeasurements are normally distributed. The intervals estimated with the method proposed in this paper correspond to the 0.13 and 99.87 percentiles, while the expanded uncertainties obtained with the WLS are obtained from the standard uncertainties with coverage factor 3 (to define the same confidence level). The estimated uncertainties are clearly non-symmetric and quite different from the WLS ones, thus reflecting the intrinsic non-gaussianity of the quantities involved in the estimation process.

The performance are also verified in terms of RMSE of the active power estimations on 300 different operative conditions, as above. Fig. 10 reports the results and shows that the proposed method generally outperforms WLS when the same measurement configuration is used. A maximum RMSE reduction is obtained for node 2 (about $22 \%$ ), while node 5 accuracy is practically unaffected, mainly due to the aforementioned associated load behavior. It is important to recall that the WLS uses the averages and the standard deviations of the available prior PDFs as pseudomeasurements. Besides, for a fair comparison, the WLS has been modified to include the covariances $\sigma_{P_{i}, Q_{i}}$ (obtained from prior information) of each node $i$ in its weighting matrix (as in [30], [31]) and all the measurements are modeled in the same way. To provide a broader picture, in Fig. 10 the results are compared also to those achievable with the weighted least absolute value (WLAV) estimation. As in the WLS, the WLAV weights are obtained from the standard deviations of the prior PDFs. The WLAV shows a lower estimation accuracy: a deeper discussion of these aspects can be found, for instance, in [32].

Finally, some considerations on the computation time can be reported, even though an optimization of the proposed algorithm for prototyping is beyond the scope of the paper. The computation time mainly depends on the measurement computation function and on the number of the points for the M-H chain. On a Windows 10 notebook with an Intel i7 $2.60 \mathrm{GHz}$ processor, under Matlab 2015 environment, with the same measurement configuration as in the last test and 500000 computed points, the computation time is about $145 \mathrm{~s}$. Even if clearly higher than classical WLS and WLAV 


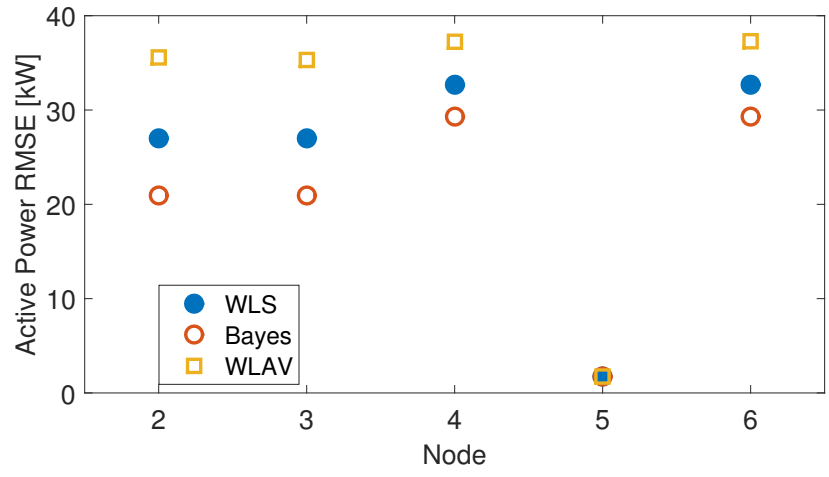

Fig. 10. Comparison of active power estimation RMSE by means of Bayes, WLS and WLAV approaches

estimators (ranging from a few to hundreds of milliseconds in similar conditions) such time durations are perfectly compatible with the typical update interval of DMSs and load profile information, which operate on a 15 minutes basis. It is interesting to notice that even for larger networks the number of points does not suffer of the dimensionality curse of classical Monte Carlo methods and combinatorial approaches [33]. For instance, with the 16-bus network used in [20], the computation time increases of less than $10 \%$. For very large networks, a multi-area approach (as in [34]) can be easily integrated with the proposed method. When correlation is introduced the overall results are marginally affected and, as expected, correlation helps in dimensionality reduction, thus allowing good performance also with less points.

\section{CONCLUSiON}

The paper presents a DSSE based on Bayes approach that is able to include every type of measurement and pseudomeasurement, no matter what is their statistical model. In particular, the proposed DSSE is able to reflect the dependency between active and reactive power and to directly translate prior information on the power behavior of customers/generators. The algorithm can be applied to MV networks and allows achieving accurate estimations of the power flows, while giving also a rich description of the uncertainty associated with the estimated quantities, thus making it suitable to be employed in the control applications relying on the DSSE outcomes.

\section{REFERENCES}

[1] J. Fan and S. Borlase, "The evolution of distribution," Power and Energy Magazine, IEEE, vol. 7, no. 2, pp. 63-68, March 2009.

[2] G. Heydt, "The next generation of power distribution systems," IEEE Trans. Smart Grid, vol. 1, no. 3, pp. 225-235, Dec. 2010.

[3] M. Baran and A. Kelley, "A branch-current-based state estimation method for distribution systems," IEEE Trans. Power Syst., vol. 10, no. 1, pp. 483-491, Feb. 1995.

[4] W. M. Lin and J. H. Teng, "Distribution fast decoupled state estimation by measurement pairing," Generation, Transmission and Distribution, IEE Proceedings-, vol. 143, no. 1, pp. 43-48, 1996.

[5] M. Pau, P. A. Pegoraro, and S. Sulis, "Efficient branch-current-based distribution system state estimation including synchronized measurements," IEEE Trans. Instrum. Meas., vol. 62, no. 9, pp. 2419-2429, Sep. 2013.
[6] D. A. Haughton and G. T. Heydt, "A linear state estimation formulation for smart distribution systems," IEEE Trans. Power Syst., vol. 28, no. 2, pp. 1187-1195, May 2013.

[7] A. Cataliotti, V. Cosentino, D. D. Cara, and G. Tinè, "LV measurement device placement for load flow analysis in MV smart grids," IEEE Trans. Instrum. Meas., vol. 65, no. 5, pp. 999-1006, May 2016.

[8] D. Della Giustina, M. Pau, P. A. Pegoraro, F. Ponci, and S. Sulis, "Electrical distribution system state estimation: measurement issues and challenges," IEEE Instrum. Meas. Mag., vol. 17, no. 6, pp. 36-42, Dec. 2014.

[9] A. Primadianto and C. N. Lu, "A review on distribution system state estimation," IEEE Transactions on Power Systems, 2016, online preprint.

[10] A. Abur and A. G. Expòsito, Power System State Estimation. Theory and Implementation. Marcel Dekker, New York, 2004.

[11] A. Angioni, T. Schlösser, F. Ponci, and A. Monti, "Impact of pseudomeasurements from new power profiles on state estimation in lowvoltage grids," IEEE Trans. Instrum. Meas., vol. 65, no. 1, pp. 70-77, Jan 2016.

[12] Q. Chen, D. Kaleshi, Z. Fan, and S. Armour, "Impact of smart metering data aggregation on distribution system state estimation," IEEE Transactions on Industrial Informatics, vol. 12, no. 4, pp. 1426-1437, Aug 2016.

[13] F. Adinolfi, F. D’Agostino, A. Morini, M. Saviozzi, and F. Silvestro, "Pseudo-measurements modeling using neural network and fourier decomposition for distribution state estimation," in IEEE PES Innovative Smart Grid Technologies, Europe, Oct 2014, pp. 1-6.

[14] M. B. D. C. Filho, J. C. S. de Souza, and M. T. Schilling, "Generating high quality pseudo-measurements to keep state estimation capabilities," in 2007 IEEE Lausanne Power Tech, July 2007, pp. 1829-1834.

[15] A. Ghosh, D. Lubkeman, M. Downey, and R. Jones, "Distribution circuit state estimation using a probabilistic approach," IEEE Trans. Power Syst., vol. 12, no. 1, pp. 45-51, 1997.

[16] S. W. Heunis and R. Herman, "A probabilistic model for residential consumer loads," IEEE Transactions on Power Systems, vol. 17, no. 3, pp. 621-625, Aug 2002.

[17] R. Singh, B. C. Pal, and R. A. Jabr, "Statistical representation of distribution system loads using gaussian mixture model," IEEE Trans. Power Syst., vol. 25, no. 1, pp. 29-37, Feb 2010.

[18] G. Valverde, A. T. Saric, and V. Terzija, "Stochastic monitoring of distribution networks including correlated input variables," IEEE Trans. Power Syst., vol. 28, no. 1, pp. 246-255, Feb. 2013.

[19] R. Mínguez, A. J. Conejo, and A. S. Hadi, Non Gaussian State Estimation in Power Systems. Boston: Birkhäuser Boston, 2008, pp. 141-156. [Online]. Available: http://dx.doi.org/10.1007/ 978-0-8176-4626-4_10

[20] A. Angioni, M. Pau, F. Ponci, A. Monti, C. Muscas, S. Sulis, and P. A. Pegoraro, "Bayesian distribution system state estimation in presence of non-gaussian pseudo-measurements," in 2016 IEEE International Workshop on Applied Measurements for Power Systems (AMPS), Sept 2016, pp. 1-6.

[21] R. Singh, E. Manitsas, B. C. Pal, and G. Strbac, "A recursive bayesian approach for identification of network configuration changes in distribution system state estimation," IEEE Transactions on Power Systems, vol. 25, no. 3, pp. 1329-1336, Aug. 2010.

[22] "Evaluation of data - guide to the expression of uncertainty in measurement," JCGM 100:2008, Sep. 2008.

[23] J. M. Bernardo and A. F. M. Smith, Bayesian Theory. Wiley, Chichester, U.K., 2004.

[24] C. Muscas, M. Pau, P. A. Pegoraro, and S. Sulis, "Uncertainty of voltage profile in pmu-based distribution system state estimation," IEEE Trans. Instrum. Meas., vol. 65, no. 5, pp. 988-998, May 2016.

[25] R. B. Nelsen, An Introduction to Copulas. Springer, 1999.

[26] H. Haario, E. Saksman, and J. Tamminen, "Adaptive proposal distribution for random walk metropolis algorithm," Computational Statistics, vol. 14, pp. 375-395, Aug. 1999.

[27] OpenEI open energy information. [Online]. Available: http://en.openei. org/datasets/files/961/pub/

[28] NREL. PVWatts Calculator tool. [Online]. Available: http://pvwatts. nrel.gov

[29] ATLANTIDE project. [Online]. Available: http://www.progettoatlantide. it/

[30] M. Asprou, E. Kyriakides, and M. Albu, "The effect of variable weights in a wls state estimator considering instrument transformer uncertainties," IEEE Trans. Instrum. Meas., vol. 63, no. 6, pp. 14841495, Jun. 2014. 
[31] C. Muscas, M. Pau, P. Pegoraro, and S. Sulis, "Effects of measurements and pseudomeasurements correlation in distribution system state estimation," IEEE Trans. Instrum. Meas., vol. 63, no. 12, pp. 2813-2823, Dec. 2014.

[32] R. Singh, B. Pal, and R. Jabr, "Choice of estimator for distribution system state estimation," IET Gener. Transm. Distrib., vol. 3, no. 7, pp. 666-678, Jul. 2009.

[33] C. P. Robert and G. Casella, Introducing Monte Carlo Methods with R. Springer, New York, NY, 2010

[34] M. Pau, F. Ponci, A. Monti, S. Sulis, C. Muscas, and P. A. Pegoraro, "An efficient and accurate solution for distribution system state estimation with multiarea architecture," IEEE Transactions on Instrumentation and Measurement, vol. 66, no. 5, pp. 910-919, May 2017.

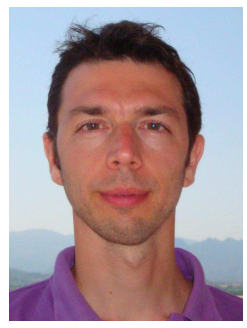

Paolo Attilio Pegoraro (M'06) received the M.S. (summa cum laude) degree in telecommunications engineering and the Ph.D. degree in electronic and telecommunications engineering from the University of Padova, Padova, Italy, in 2001 and 2005, respectively.

He is currently an Assistant Professor of Electrical and Electronic Measurements with the Department of Electrical and Electronic Engineering, University of Cagliari, Cagliari, Italy and, in 2017, received the National Academic Qualification as Associate

Professor.

He has authored or co-authored over 70 scientific papers. His current research interests include the development of new measurement techniques for modern power networks, with particular attention to synchronized measurements and state estimation for distribution grids.

Dr. Pegoraro is a member of the IEEE Instrumentation and Measurement Society and of the TC-39 - Measurements in Power Systems.

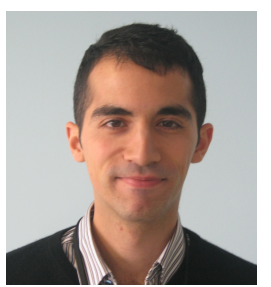

Andrea Angioni (S'13) received the M.Sc. degree in electrical engineering from the University of Cagliari, Cagliari, Italy, in 2013. He joined the Institute for Automation of Complex Power Systems, E.ON Energy Research Center, RWTH Aachen University, Aachen, Germany, in 2013. His current research interests include automation systems for distribution networks with a focus on monitoring systems and state estimation.

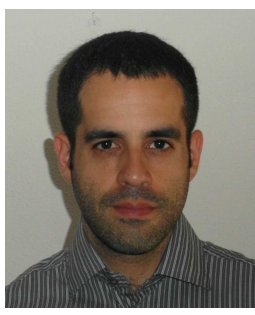

Marco Pau (S'14-M'15) received the M.S. (cum laude) degree in electrical engineering and the Ph.D. degree in electronic engineering and computer science from the University of Cagliari, Italy, in 2011 and 2015, respectively.

Currently, he is a research associate at the Institute for Automation of Complex Power Systems at the E.ON Energy Research Center, RWTH Aachen University, where he is leader of the team for Power System Automation.

His main research activity concerns the design of measurement systems applied to electric distribution systems and devised for distribution grid automation.

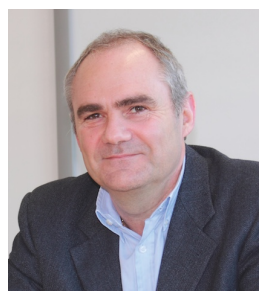

Antonello Monti (M'94-SM'02) received his M.Sc degree (summa cum laude) and his $\mathrm{PhD}$ in Electrical Engineering from Politecnico di Milano, Italy in 1989 and 1994 respectively.

He started his career in Ansaldo Industria and then moved in 1995 to Politecnico di Milano as Assistant Professor. In 2000 he joined the Department of Electrical Engineering of the University of South Carolina (USA) as Associate and then Full Professor. Since 2008 he is the director of the Institute for Automation of Complex Power System within the E.ON Energy Research Center at RWTH Aachen University.

Dr. Monti is author or co-author of more than 300 peer-reviewed papers published in international Journals and in the proceedings of International conferences. He is a Senior Member of IEEE, Associate Editor of the IEEE System Journal and Associate Editor of IEEE Electrification Magazine. Dr. Monti is the recipient of the 2017 IEEE Innovation in Societal Infrastructure Award.

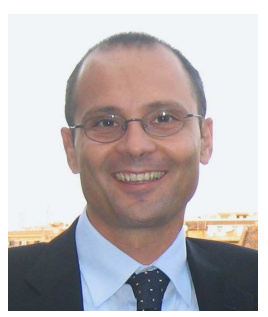

Carlo Muscas (M'98-SM15) received the M.S (cum laude) degree in electrical engineering from the University of Cagliari, Cagliari, Italy, in 1994.

$\mathrm{He}$ was Assistant Professor with the University of Cagliari from 1996 to 2001. Since 2001 he has been an Associate Professor of Electrical and Electronic Measurement with the University of Cagliari, where he is currently the Chairman of the Council for the B.S. degree in Electrical, Electronic and Computer Engineering.

His current research interests include the measurement of synchronized phasors, the implementation of distributed measurement systems for modern electric grid, and the study of power quality phenomena $\mathrm{He}$ has authored and co-authored more than 130 scientific papers.

Mr. Muscas is currently an Associate Editor of the IEEE TRANSACTIONS ON INSTRUMENTATION AND MEASUREMENT and is the Chairman of TC 39 (Measurements in Power Systems) of IEEE Instrumentation and Measurement Society.

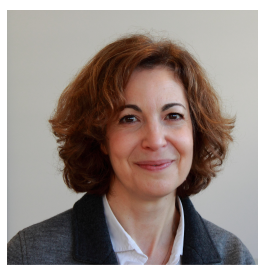

Ferdinanda Ponci (M00SM08) graduated with $\mathrm{Ph} . \mathrm{D}$. in Electrical Engineering from the Politecnico di Milano, in 2002.

She joined the Department of Electrical Engineering, University of South Carolina, as an Assistant Professor in 2003, and became Associate Professor in 2008. In 2009, she joined the Institute for Automation of Complex Power Systems, RWTH Aachen University, where she is currently Professor for Monitoring and distributed control for power systems.

She is Senior Member of IEEE, and of the AdCom of the IEEE Instrumentation and Measurement Society.

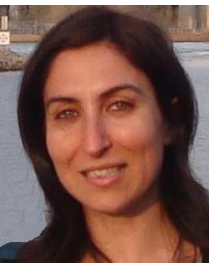

Sara Sulis (M'06) received the M.S. degree in electrical engineering and the Ph.D. degree in industrial engineering from the University of Cagliari, Cagliari, Italy, in 2002 and 2006, respectively. In 2013, she received the Habilitation to the position of Associate Professor. She is currently an Assistant Professor of Electrical and Electronic Measurements with the University of Cagliari.

She has authored or co-authored more than $80 \mathrm{sci}-$ entific papers. Her current research interests include Power Quality issues and distributed measurement systems designed to perform state estimation and harmonic sources estimation of distribution networks.

Dr. Sulis is a member of the IEEE Instrumentation and Measurement Society and the TC-39-Measurements in Power Systems. 